\section{Turning off GTP signals}

THE discovery of GAP activities in targets of the heterotrimeric $\mathrm{G}$ proteins brings them into step with their cousins in the GTPase superfamily ${ }^{1}$, the Ras-like monomeric GTPases and the initiation and elongation factors of ribosomal protein synthesis. Of these, bacterial elongation factor EF-Tu has been subjected to the most intensive scrutiny. Aminoacyl transfer RNA (aa-tRNA) binds selectively to Tu.GTP, but does not accelerate its very slow intrinsic GTPase activity. Through binding of its anticodon to the mRNAprogrammed ribosome, however, aa-tRNA does position Tu.GTP for interaction with a GAP located in the ribosome's 50 S subunit; very quickly (within a few milliseconds), the ribosomal GAP triggers hydrolysis of Tu-bound GTP.

It is instructive to compare protein complexes that regulate GTPase activities of different members of the superfamily. Each GTPase in its unadorned G. GTP state ( $a$ in the figure) hydrolyses GTP relatively slowly (tens of seconds for $\alpha$ GTP, many minutes for Tu.GTP). In most cases ( $b$ in the figure) the physiologically relevant and much faster turn-off switch is thrown when G.GTP forms a complex with other components $-\alpha_{\mathrm{g}}+$ phospholipase $\mathrm{C}$ (PLC)- $\beta 1, \alpha_{\mathrm{t}} \cdot \mathrm{GTP}+\mathrm{PDE}-\alpha \beta+\mathrm{PDE}-\gamma$, or Tu.GTP + aa-tRNA + ribosome. Just as the $\gamma$-subunit of the PDE holoenzyme can suffice to stimulate the GTPase of $\alpha_{t}$, ribosomes by themselves (at high concentrations) can stimulate GTP hydrolysis by EF-Tu. Although neither aa-tRNA nor PDE- $\alpha \beta$ stimulates GTP hydrolysis, both of these non-GAP elements serve to regulate the GAP - by inhibiting GAP in the presence of cGMP (PDE- $\alpha \beta$ ) or by guiding Tu.GTP to the GAP (aa-tRNA).

Despite these and other differences, the prevailing theme is the same: although the GAP by itself can act on G.GTP, physiological turn-off of G.GTP occurs when it binds simultaneously to its target molecule and to its GAP. Indeed PLC- $\beta 1$, a single molecule, acts as both the effector target and the GAP for $\alpha_{\mathrm{q}} \cdot$ GTP.

Two other models merit consideration. First (as in $c$ in the figure), the
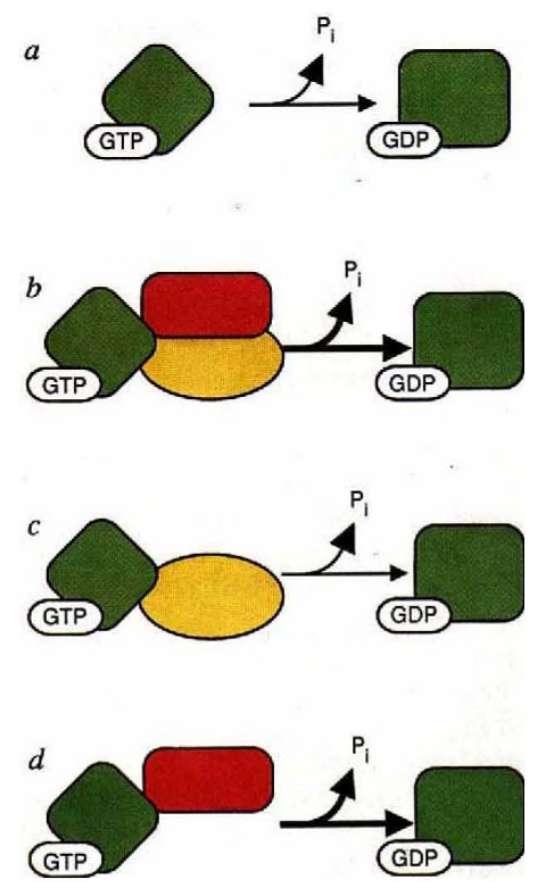

target is not associated with a GAP activity. The example of aa-tRNA and Tu.GTP shows that a target molecule may bind to a GTPase without accelerating hydrolysis of bound GTP. Some of the many isozymes of hormone-sensitive adenylyl cyclase may belong to this category. In fact, the rates at which adenylyl cyclase activities decay after hormonal stimulation $^{19,20}$ agree rather well with measured rates of GTP hydrolysis by pure $\alpha_{\mathrm{s}}^{21}$.

The second model ( $d$ in the figure) involves a putative 'killer-GAP' that is not associated with an effector target. Although they normally function as GAPs in association with specific target molecules, PDE- $\gamma$ and the ribosome show that it is possible to deactivate the GTP-bound form of a protein in the absence of its target. At least two proteins, Ras-GAP and neurofibromin, can trigger the GTPase activity of p21 ${ }^{\text {ras }}$, and GAPs have been identified for many other members of the expanding family of monomeric small GTPases (for review, see ref. 22). Are these killer-GAPs or do they also act as (or in association with) effector targets? Despite generating a number of ingenious experiments, the debate over these alternatives ${ }^{22-24}$ has come to no firm conclusion, for a very simple reason: we are in no position to determine the relation between GAPs and effectors, because no example of the latter is in hand. Despite the many well-studied cellular consequences of activating $p 21^{\text {ras }}$, we cannot point to a single molecule (or even a biochemical activity) that is directly regulated by the GTP-bound form of this GTPase (or of other Ras-like proteins). To speak plainly, we do not even know whether $\alpha$.GTP or EF-Tu is the appropriate model for the action of $\mathrm{p} 21^{\text {ras }}$ - that is, whether the protein works by regulating the biochemical activity of a target protein or by regulating the assembly of separate components into a macromolecular complex.

Despite our ignorance, the discovery of GAP activities associated with effector targets of G-protein $\alpha$-subunits furnishes a persuasive analogy. The new observations strongly support the view that some of the GAPs for $\mathrm{p} 21^{\text {ras }}$ and its siblings are effectors (by analogy with PLC- $\beta 1$ and $\alpha_{\mathrm{q}}$ ), whereas others (by analogy with PDE- $\gamma$ and $\alpha_{\mathrm{t}}$ ) act in association with effector target proteins. The likelihood that $\mathrm{p} 21^{\text {ras }}$ or other monomeric GTPases associate only with killer-GAPs now appears to be vanishingly small.

H.R.B. \& L.S. stimulated PDE activity turning off at the same rapid rate, even in the presence of a high concentration of cGMP. Because both sets of results are convincing and internally consistent, it is not clear why cGMP should slow GTPase activity in one $\operatorname{case}^{7}$ but fail to prolong PDE activity in the other ${ }^{13}$.)

The exciting discovery that effectors of $G$ proteins also act as GAPs for $G$ proteins carries broad implications for understanding other proteins in the GTPase superfamily, including the ever enigmatic p21 ras (see box). This discovery also forces us to modify the prevailing model of how signalling $G$ proteins work. In that model, the slow turn-off of $\alpha \cdot$ GTP, mediated by its intrinsic GTPase activity, seemed to account for the ability of $G$ proteins to amplify hormone signals. Now we realize that fixing the spotlight on the prima ballerina gave predicted turn-off rates that were far too slow for physiology - a difficulty that is neatly solved by converting the turn-off into a pas de deux. Association of GAP activities with effectors preserves amplification of signals, but to a degree determined by the effector rather than by the G-protein $\alpha$-subunit. Furthermore, the GAP activity can itself be regulated and it may vary among different effectors, thus further enhancing the versatility and flexibility of signals mediated by $\mathrm{G}$ proteins. It would not be surprising to learn, for example, that some of the many recently discovered adenylyl cyclase isozymes (see ref. 14 for summary) differ primarily in their abilities to act as GAPs for the $\alpha$-subunit of $\mathrm{G}_{\mathrm{s}}$, the stimulatory regulator of adenylyl cyclase.

Finally, we should recognize that sometimes extinction of a G-proteinmediated response may require more than the GAP activity of an effector. Until now we have assumed that only one $\alpha \cdot$ GTP can activate each effector, so that the effector's GAP activity effectively turns off the response. What if an activated receptor generates a number of $\alpha$.GTP molecules greater than the number of effectors available to turn them off? Indeed, an excess of $\mathrm{G}$ proteins over effectors may be in some cases a 